\title{
Magnetic Levitation of Diamond Modified with Manganese and Bismuth
}

\author{
Takahisa Yamazaki, Amane Muraoka \\ School of Engineering, Tokyo Institute of Technology, Tokyo, Japan \\ Email: yamazaki.t.ae@m.titech.ac.jp
}

How to cite this paper: Yamazaki, T. and Muraoka, A. (2021) Magnetic Levitation of Diamond Modified with Manganese and Bismuth. Materials Sciences and Applications, 12, 78-88.

https://doi.org/10.4236/msa.2021.121005

Received: December 4, 2020

Accepted: January 19, 2021

Published: January 22, 2021

Copyright $\odot 2021$ by author(s) and Scientific Research Publishing Inc. This work is licensed under the Creative Commons Attribution International License (CC BY 4.0).

http://creativecommons.org/licenses/by/4.0/

\begin{abstract}
Operating diamond grits to produce a precise grind tool is need. In order to lift up a diamond grit by magnetic force, the magnetic flux was estimated to be $100 \mu \mathrm{T} / \mathrm{g}$. Diamond (110) surface was modified with manganese powder at $450^{\circ} \mathrm{C}(720 \mathrm{~K})$ then with bismuth powder at $270^{\circ} \mathrm{C}(540 \mathrm{~K})$ due to its low melting temperature. Manganese carbides were formed on the diamond surface which was confirmed by an X-ray diffraction. A magnet that exhibits ferrimagnetism was formed on the surface, it had a spontaneous magnetism. We conducted to form six small disk magnets at hexagonal apex positions on the diamond (110) by using gold film mask. The magnetic flux measured at the center of the hexagon magnets on was $232 \mu \mathrm{T}$ at room temperature, and the surface modified diamond grid could be lifted up in the magnetic field between two solenoid coils.
\end{abstract}

\section{Keywords}

Manganese Carbide, MnBi, Magnet, Levitation, Diamond

\section{Introduction}

As known anti ferrous magnetism of $\mathrm{MnO}$, manganese compounds exhibit various magnetic properties. The manganese compounds of $\mathrm{MnAs}$ and $\mathrm{MnBi}$ are known as ferrimagnetism materials [1]. P. Karen et al. [2] reported the manganese carbide $\mathrm{Mn}_{7} \mathrm{C}_{3}$ and $\mathrm{Mn}_{5} \mathrm{C}_{2}$ microstructure and paramagnetic property. On the other hand, bismuth exhibits diamagnetism. Manganese and carbon react to be formed carbide easily. A mixture of a fine diamond powder and manganese powder reacted not to remain the diamond powder. Infrared ray heating has less damage to diamond due to the transparency into diamond. The three surfaces of (111), (100) and (110) of diamond were attempted to be surface modification 
using diamond powder by infrared ray irradiation [3] [4]. Diamond (110) was modified with manganese powder by infrared ray irradiation to form lamellar manganese carbide. An alloy of MnBi has been utilized as permanent magnet under room temperature. The deposited films of $\mathrm{MnBi}$ on glass or mica by fabricated by vapor deposition exhibit magneto-optical effect. The deposition series were manganese then bismuth to form epitaxial growth of an ally of $\mathrm{MnBi}$ [5]. When the composite of $\mathrm{MnBi}$ are formed on diamond (110), the magnetic flux would be more than the manganese carbides. We propose thin MnBi magnets on lamellar manganese carbide modified the (110) surface of the diamond using infrared ray heating.

Six disk magnets at the sites of hexagonal apex on the diamond surface were conducted to be formed in order to obtain a high density magnetic flux at the center of the hexagonal magnets than each magnet. This method was used in levitation technology to slide precisely [6].

Magnetic flux was measured by Hall effect probe. The probe was utilized as a positioning sensor with magnets. Using Hall effect sensor at the edge of a solenoid coil, the modified diamond would be lifted up stably at level.

\section{Experimental Procedures}

\subsection{Differential Thermal Analysis for the Magnet Formation Process}

Manganese and Bismuth were materials which did not damage the diamond at a temperature with a rage where the $\mathrm{Mn}$-Bi alloys were formed although the manganese reacts diamond quickly. Differential thermal analysis (DTA) for a diamond powder was conducted to confirm a magnet formation process by using manganese and bismuth. A commercially available diamond powder passed through 30 $\mu \mathrm{m}$ sieve grids was used. Manganese powder and manganese powder for DTA test were produced respectively by a ball mill using alumina balls for hour to be a sample of $45 \mu \mathrm{m}$ or less in diameter. These powders were mixed at a molar ratio of C:Mn:Bi = 3:7:1, based on the molar ratio to form $\mathrm{Mn}_{7} \mathrm{C}_{3}$ and $\mathrm{MnBi}$. The mixed powder with $30 \mathrm{mg}$ in mass were placed in a platinum cell of DTA/DTG sensor. This cell and sample alumina cell were heated from $30^{\circ} \mathrm{C}$ to $800^{\circ} \mathrm{C}(300 \mathrm{~K}$ to $1070 \mathrm{~K}$ ) at the heating rate of $10 \mathrm{~K} / \mathrm{min}$ with Argon gas flowed at $60 \mathrm{ml} / \mathrm{min}$.

\subsection{Magnet Formation on Diamond (110)}

Surface modification of diamond (110) with Mn-Bi by infrared ray heating was carried out in vacuum to prevent oxidation in order to minimize carbon desorption from diamond surface. Artificial diamond of half cut diamond of $2 \mathrm{~mm}$ in thickness was used. The mass of the diamond was $0.069 \mathrm{~g}$. The diamond surface (110) was manufactured by a laser cutting in half cut of the diamond (100). Another rectangular disk shape of diamond was prepared. The dimension was $3.0 \times 2.5 \times 1.0 \mathrm{~mm}$. The mass was $0.030 \mathrm{~g}$. The diamond surface was covered with gold spattering film preventing the reaction of manganese except six spots. 
Gold spattering by argon ions was carried out for $10 \mathrm{~min}$ to become a film of 30 $\mu \mathrm{m}$ in thickness. The gold film on diamond (110) was removed off at 6 spots where were the vertexes of the regular hexagon. The spot area was the circle with a diameter of $0.5 \mathrm{~mm}$ which was corresponding space for surface modification by manganese powder and bismuth powder as shown in Figure 1. The regular circle spots were produced by FIB (Focused Ion Beam) fabrication using Ga ion spattering. The specimen of diamond (110) with gold spattering film and appearance after FIB fabrication are shown in Figure 2(a) and Figure 2(b). Manganese powder and bismuth powder were produced as mentioned before was mounted on diamond surface (110) at a selected molar ratio. The weight of manganese was $8.0 \mathrm{mg}$ and it of bismuth was $4.4 \mathrm{mg}$. The purity of the manganese powder was 98.0 and it of the bismuth powder was 99.999 .

Infrared rays emitted by a halogen lamp are focused by gold elliptical mirror. The condensed rays were passed through fused quartz window into a vacuum chamber. The irradiation area on the sample was $20 \mathrm{~mm}$ in diameter. The chamber was evacuated by turbo molecular drag pump under $8.0 \times 10^{-4} \mathrm{~Pa}$. Firstly, manganese powder was mounted on diamond and heated at $450^{\circ} \mathrm{C}(720 \mathrm{~K})$ to form manganese carbides for $10 \mathrm{~s}$. Then bismuth powder was mounted on the manganese powder and heated at $270^{\circ} \mathrm{C}(540 \mathrm{~K})$ for $120 \mathrm{~s}$ as shown in Figure 3.

\subsection{Measurement of Magnetic Flux}

Low temperature Hall effect sensor was used. In order to measure the magnetic flux density, geomagnetism was taken as $36 \mu \mathrm{T}$ in Tokyo in vertical component. The measurement voltage was $2.52 \times 10 \mathrm{mV}$. The coefficient was $0.7 \times 10^{-2} \mathrm{~V} / \mathrm{T}$, and the operating temperature range specification was from 4 to $373 \mathrm{~K}$. The Hall element was protected by two electrical resistances of $50 \mathrm{~W}$ and the output signal was amplified by the DC amplifier. The measurement temperatures were room temperature and the measurement value of the magnetic flux at every spot was obtained as shown in Figure 3. The distance from a magnet to the sensor was constant by the flat ceramic cover of the sensor.

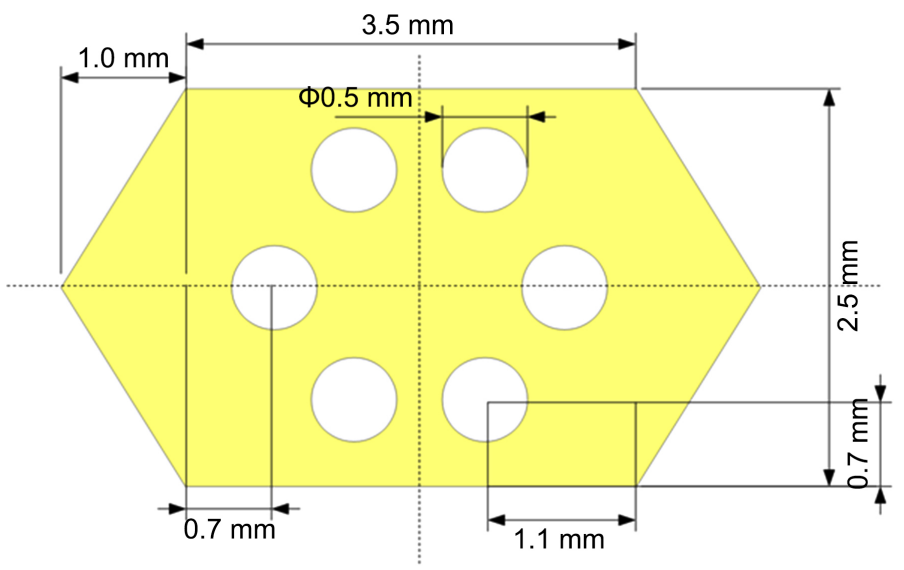

Figure 1. Dimensions of the holes of gold covered film on diamond fabricated by focused ion beam. 


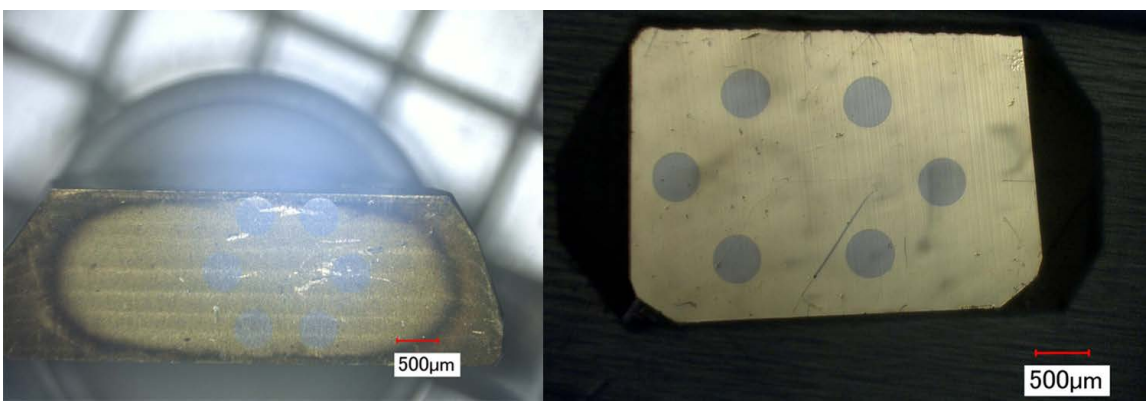

(a)

(b)

Figure 2. Artificial diamond sample images after FIB fabrication, (a) Sample 1 diamond (110); (b) Sample 2 diamond (110).

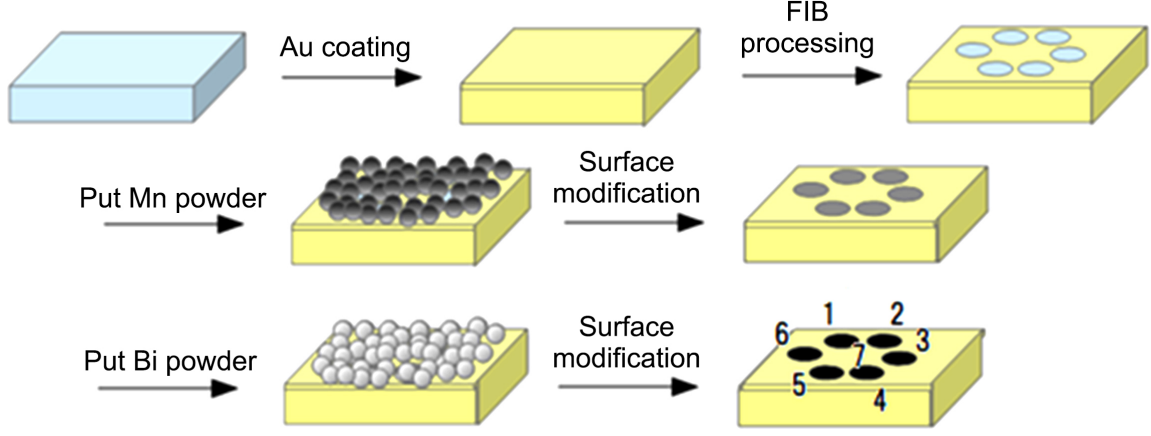

Figure 3. Procedures of surface modification using Mn powder and Bi powder on the gold coated surface of diamond.

A Vibrating Sample Magnetometer (VSM) was used to obtain a magnetization curve of the modified diamond surface. The magnetometer was equipped with the physical properties measurement system, it can apply the magnetic field of 7 $\mathrm{T}$ in horizontal direction and the measurement temperature ranges from 1.9 to $400 \mathrm{~K}$. For the diamond specimens, the magnetization-magnetic field curves were measured at $10 \mathrm{~K}$ varied magnetic field from -4000 to $4000 \mathrm{kA} / \mathrm{m}$.

\section{Results}

\subsection{Thermal Analysis for Mixed Powder}

DTA/DTG curves for the mixed powder are shown in Figure 4. Solid line shows DTA curve and dotted line shows DTG curve. Endothermal peak can be observed at $276^{\circ} \mathrm{C}(550 \mathrm{~K})$. The endothermal peak was considered to be a melting point of bismuth. Other endothermal peak around $355^{\circ} \mathrm{C}(628 \mathrm{~K})$ can be observed. It indicates the phase transition from $\mathrm{MnBi}$ to $\mathrm{Mn}_{1.08} \mathrm{Bi}$ with endothermal reaction. A mark at $450^{\circ} \mathrm{C}(720 \mathrm{~K})$ is sited. One small peak at $420^{\circ} \mathrm{C}(690 \mathrm{~K})$ can be obtained. This temperature is considered to be related with the formation of $\mathrm{Mn}_{7} \mathrm{C}_{3}$ according to the binary phase diagram of $\mathrm{Mn}-\mathrm{C}$. The reason why the temperature of manganese carbide was detected is small amount of bismuth. If amount of the bismuth is large, manganese atoms will be consumed in formation of $\mathrm{MnBi}$ at $270^{\circ} \mathrm{C}(540 \mathrm{~K})$. The DTG curves increased with temperature. It 
indicates manganese and bismuth have been oxidized gradually. The degree of the increasing mass weight was greater than a sample of C:Mn:Bi $=1: 1: 1$ and the increase of the mass was not detected for a sample non-containing of Bi. The increasing of mass with increasing temperature was considered to be oxidation after Bi molten. The surface modification of diamond was conducted in vacuum without oxidation and using small amount of bismuth to depress the formation of $\mathrm{MnBi}$.

\subsection{Magnet Formation}

Surface modification of a diamond specimen coated with gold after heating at $450^{\circ} \mathrm{C}(720 \mathrm{~K})$ with $\mathrm{Mn}$ powder is shown in Figure 5 and the diamond specimen after heating at $270^{\circ} \mathrm{C}(540 \mathrm{~K})$ with Bi powder is shown in Figure 6. Manganese installed area on coated gold film and areas on six removed off gold film where diamond (110) bared look different. In the center of the regular hexagon, remaining gold film and manganese powder show black color. In the apex of the regular hexagon where the gold film was removed shows white color. It is considered to be formed manganese carbide and $\mathrm{MnBi}$.

The X-ray diffraction pattern of the modified diamond (110) is shown in Figure 7. The peak of diamond (110) at $76^{\circ}$ is detected. Peaks of pure gold and pure bismuth peaks are observed at near angles, $39^{\circ}$ and $65^{\circ}$. The peak at $26^{\circ}$ is considered to be $\mathrm{Bi}$ (012), graphite, and AuMn. Manganese carbide peak $\mathrm{Mn}_{7} \mathrm{C}_{3}$ at $39.5^{\circ}$ may be overlapped with $\mathrm{Bi}(110)$. From the result of $\mathrm{X}$-ray diffraction pattern, Bi has a lot of peaks. Their peaks are overlapped with other materials as pure gold. Focusing on the peak at $41^{\circ}$ and $50^{\circ}$ as shown in Figure 7, it can predict that MnBi was formed.

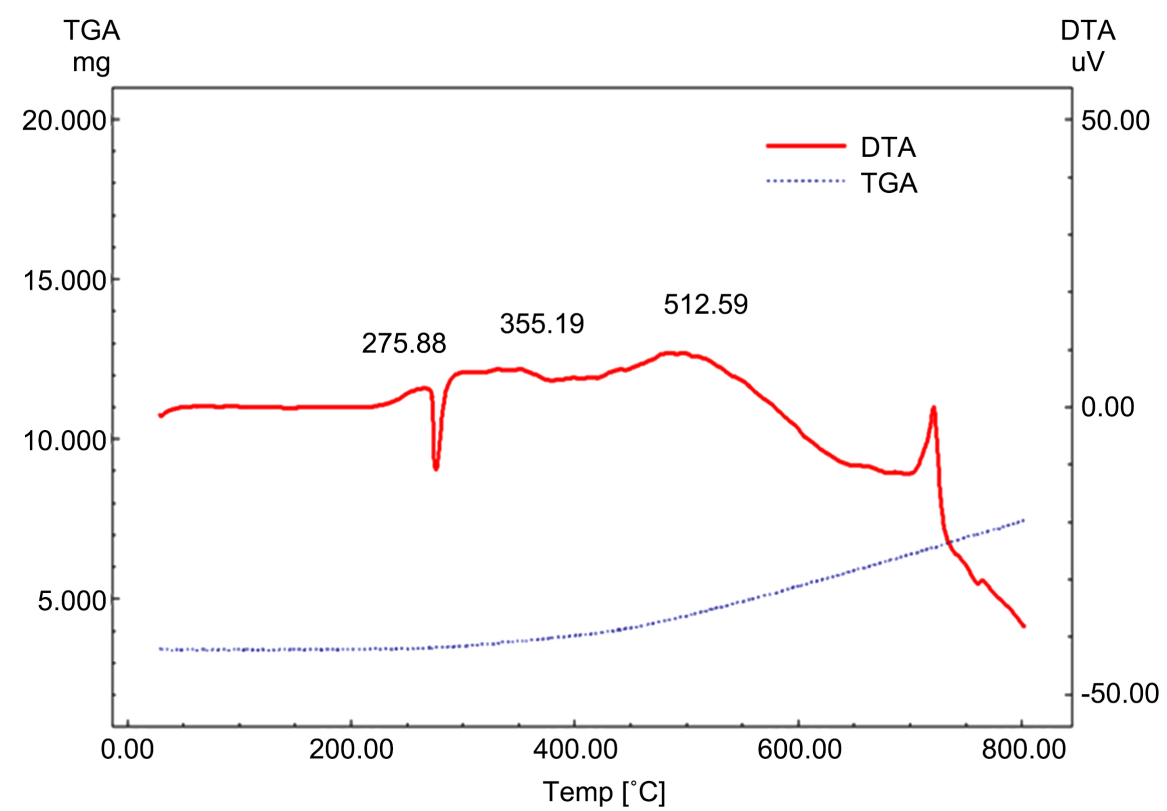

Figure 4. Differential thermal analysis curve for diamond powder and manganese powder and bismuth powder. 


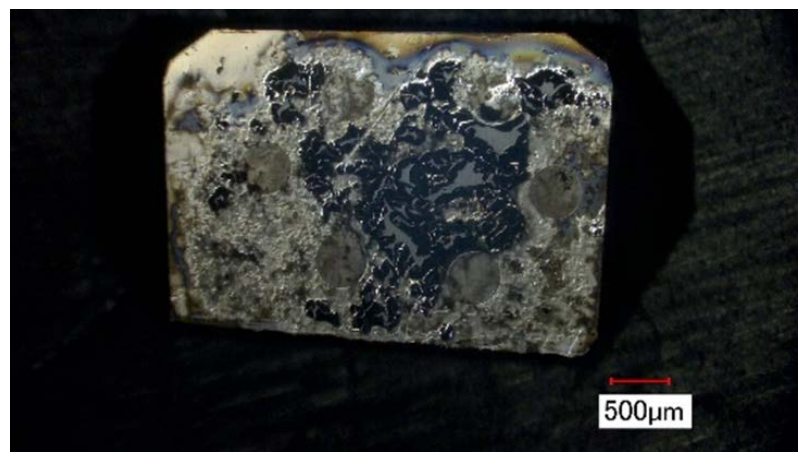

Figure 5. Optical microscope image of diamond (110) sample 2 by using manganese powder after the surface modification in the vacuum infrared ray heating furnace.

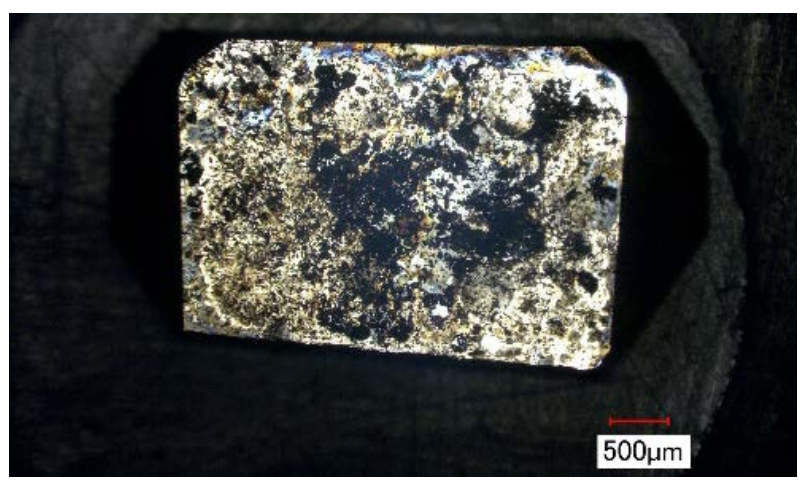

Figure 6. Optical microscope image of diamond (110) sample 2 with Bi powder after heating at $540 \mathrm{~K}\left(270^{\circ} \mathrm{C}\right)$.

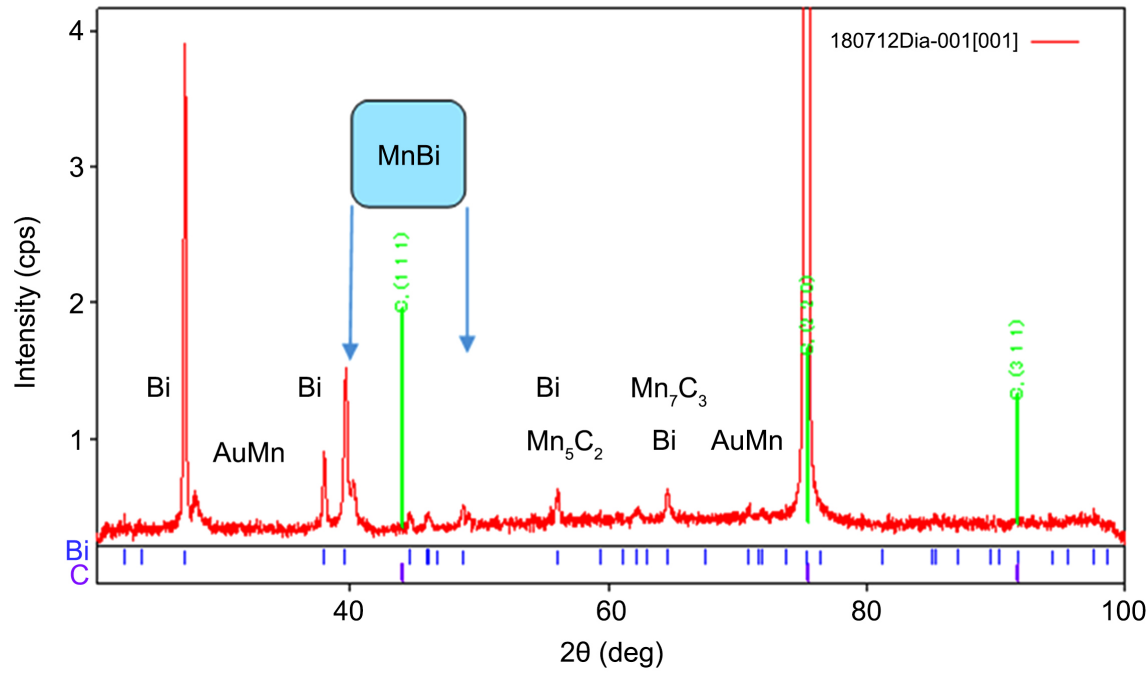

Figure 7. X-ray diffraction pattern of the modified diamond (110) with the manganese and bismuth powder.

\subsection{Evaluation of Magnetic Flux}

The measured results of magnetic flux density from the (1) to (6) formed magnets at the six apex of the regular hexagon and at the center around marked (7) as shown in Figure 3 using a Hall effect sensor were presented in Table 1. The 
measurement was conducted at room temperature. Two diamond samples of diamond (110) were different shapes and one control sample was diamond (100) that was numbered 3 . In the magnet formation process, the diamond surface coated with gold was modified by manganese powder, then the sample was measured the magnetic field by a Hall effect sensor. The values are sited in Table 1. The values of the magnetic flux density after the magnet formation process are sited at the lines of then $\mathrm{Bi}$. The values of the surface magnetic flux density became greater after the formation $\mathrm{MnBi}$ than that fabricated with only manganese powder. The small values of the sample 3: diamond (100) at the hexagon apex sites were considered to be related with the magnetization direction. The magnetization direction of $\mathrm{MnBi}$ formed on diamond (110) were ordered related to the spontaneous magnetization of $\mathrm{Mn}_{7} \mathrm{C}_{3}$. The maximum magnetic flux densities were measured near the center of the hexagonal spots, around 7 of every samples, the measured magnetic flux density was 408.7, 232.1 and $70.0 \mu$ T. Surveying with the Hall effect probe ( $1.02 \mathrm{~mm}$ in diameter) along diagonal lines of the hexagon spots revealed the center of a hexagon on diamond (110).

Figure 8 shows the magnetization curve measured for sample 2. The outer magnet applied the magnetic field to the sample from 4000 to 0 to -4000 to 4000 $\mathrm{kA} / \mathrm{m}$ at $10 \mathrm{~K}$. There is a difference of the saturated magnetization applied a magnetic field of $4000 \mathrm{kA} / \mathrm{m}$ because of the six thin magnets on the diamond (110). The Magnetic susceptibility and coercive force obtained by the VSM measurements and the value of the sample 3 obtained by SQUID are sited in Table 2. The value of $0.2 \mathrm{kA} / \mathrm{m}$ for the diamond (100) is smaller than those of diamond (110). B-H curves obtained by SQUID, the spontaneous magnetic flux was not remained. Thin layer of the manganese carbide of $\mathrm{Mn}_{7} \mathrm{C}_{3}$ on diamond (110) generated spontaneous magnetism to perpendicular axis, then the low temperature formation process formed the $\mathrm{MnBi}$ laminated layer on the $\mathrm{Mn}_{7} \mathrm{C}_{3}$. The $\mathrm{MnBi}$ layer magnetize along to the perpendicular axis due to the spontaneous magnetism of $\mathrm{Mn}_{7} \mathrm{C}_{3}$ on the diamond (110). The result of VSM revealed the ferri-magnetism of $\mathrm{Mn}_{7} \mathrm{C}_{3}$ and ferro-magnetism of $\mathrm{MnBi}$.

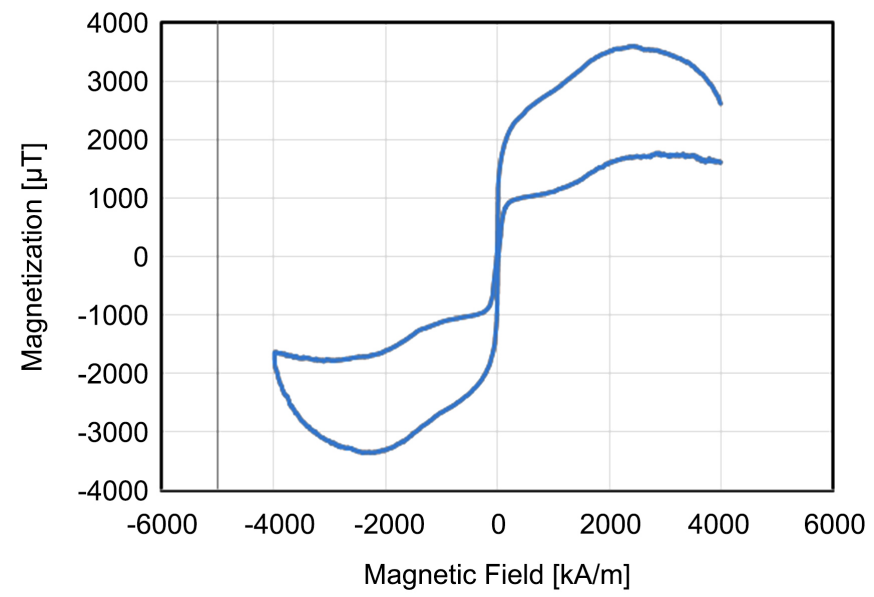

Figure 8. M-H curve of sample 2 measured at $10 \mathrm{~K}$. 
Table 1. Results of the surface magnetic flux density $[\mu \mathrm{T}]$.

\begin{tabular}{ccccccccc}
\hline Sample No. & $\begin{array}{c}\text { Modification } \\
\text { element }\end{array}$ & (1) & (2) & (3) & (4) & (5) & (6) & (7) \\
\hline 1 & Mn & 62.1 & 67.2 & 66.8 & 49 & 71.1 & 68.6 & 138.2 \\
D (110) & Then Bi & 233.2 & 218 & 293.1 & 298.5 & 258.2 & 391.4 & 408.7 \\
& & & & & & & & \\
2 & Mn & 26.4 & 31.6 & 43.4 & 24.5 & 48.8 & 61.1 & 104.1 \\
D (110) & Then Bi & 125 & 139.5 & 132.4 & 124.1 & 171.7 & 155.2 & 232.1 \\
3 cntl & Mn & 24.2 & 53.2 & 21.8 & 7.4 & 0.1 & 5.0 & 14.6 \\
D (100) & Then Bi & 43.5 & 72.4 & 82.1 & 48.3 & 45.9 & 38.7 & 70.0 \\
\hline
\end{tabular}

Table 2. Magnetic properties of each sample derived from VSM measurement results.

\begin{tabular}{cccc}
\hline Sample No. & Modified surface & $\begin{array}{c}\text { Magnetic } \\
\text { susceptibility }\end{array}$ & $\begin{array}{c}\text { Coercive force } \\
{[\mathrm{kA} / \mathrm{m}]}\end{array}$ \\
\hline 1 & $(110)$ & 2.64 & 21.2 \\
2 & $(110)$ & 2.91 & 15.0 \\
$3 \mathrm{cntl}$ & $(100)$ & 3.76 & 0.2 \\
\hline
\end{tabular}

\section{Discussion}

Higher magnetic flux on the diamond (110) modified with manganese and bismuth than manganese carbide modified surface was detected by the Hall effect element. The difference in the magnetic flux density at the locations of six hexagonal spots on modified diamond is due to the amount of manganese at the rate of $\mathrm{C}: \mathrm{Mn}: \mathrm{Bi}=3: 7: 1$. The lowest magnetic flux value after manganese modification at spot 4 among six spots was measured. The irradiation of infrared ray around spot 4 was slight difficult in the heating. In the manganese carbide formation process, manganese powder did not react with diamond then in the $\mathrm{MnBi}$ formation process, the remained manganese reacted with the bismuth to provide the ferro-magnetism.

The surface magnetic flux values in the center of the hexagonal apex magnet disk on the modified diamond (110) were enough to perform magnetic levitation of the diamonds. Figure 9 shows the flowchart of magnetic levitation with measuring magnetic force using a Hall effect sensor. Figure 10 show the photograph of the magnetic levitation of diamond sample 2 between the solenoid coils. The floating of diamond maintained by the feedback control using the sensor signal. 


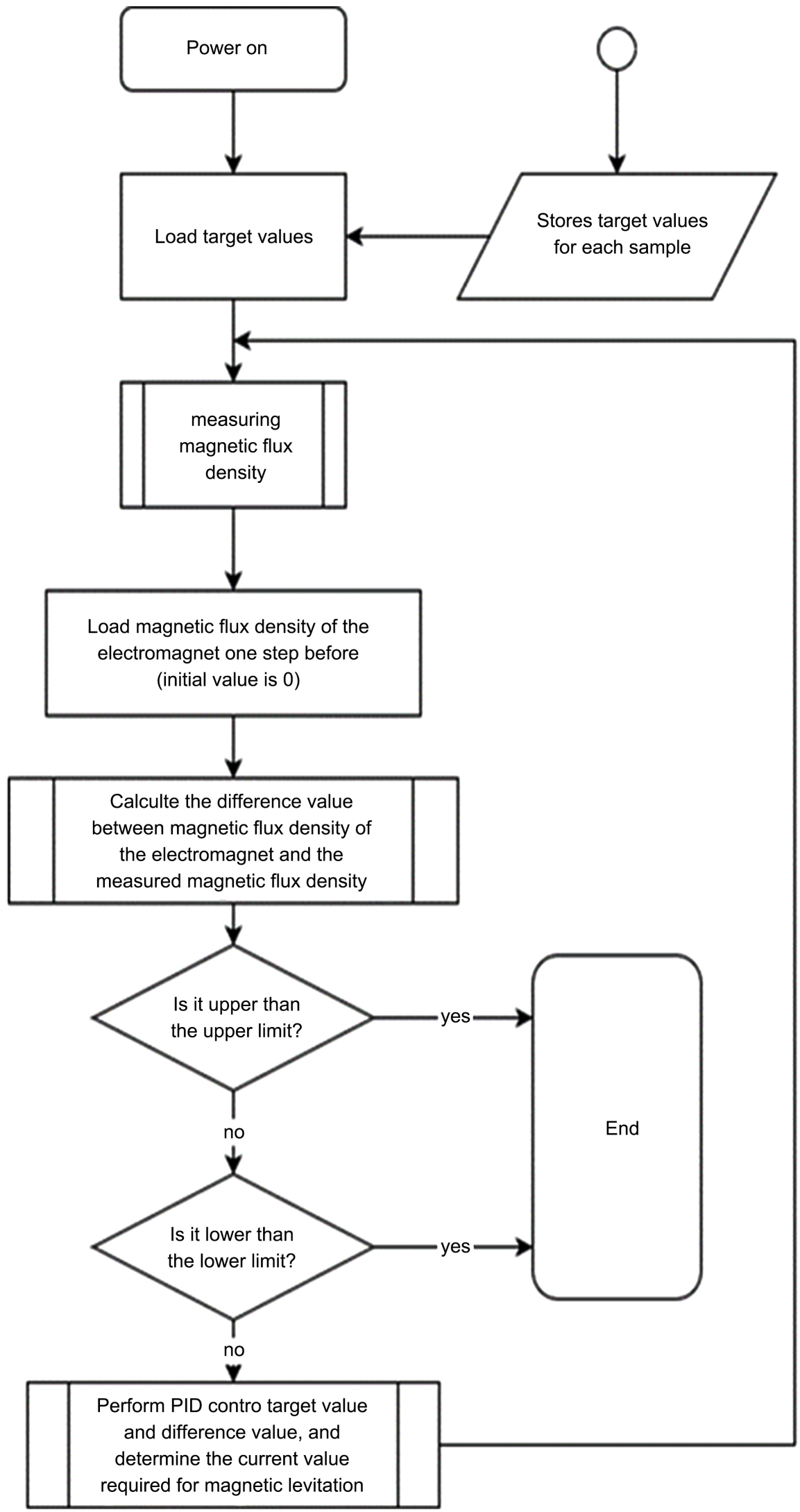

Figure 9. Flow chart of the levitation by measuring the magnetic flux by Hall effect sensor attached to upper solenoid coil. 


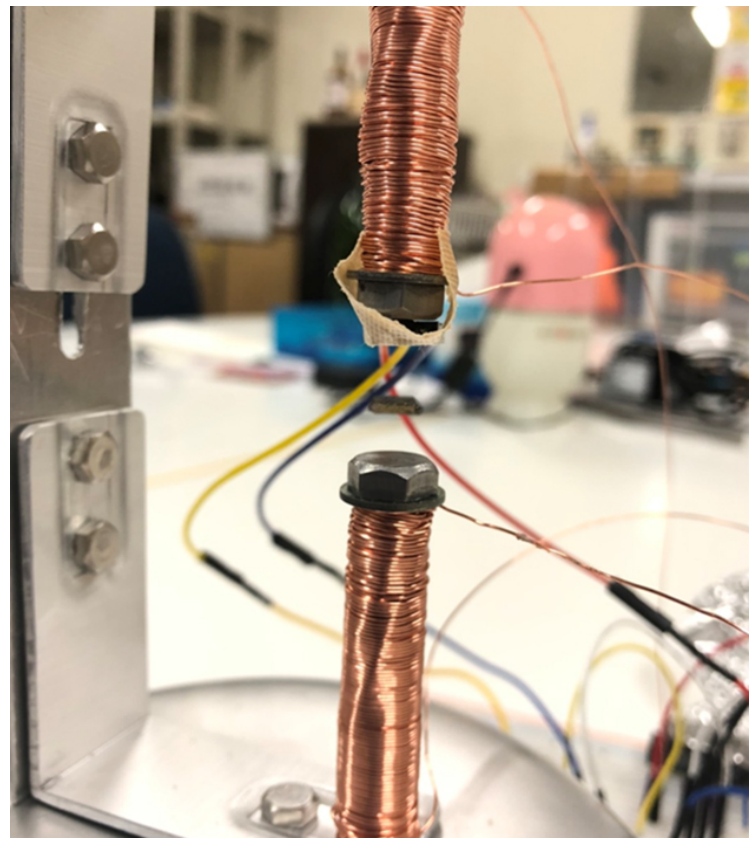

Figure 10. Magnetic levitation of the diamond sample 2 using two solenoid coils.

\section{Summary}

Diamond (110) was surface modified with manganese firstly then with bismuth to produce magnet disks. A regular hexagon magnet disks were formed using FIB process and covered diamond surface with gold. The temperature of formation of $\mathrm{MnBi}$ alloy was $270^{\circ} \mathrm{C}(540 \mathrm{~K})$, and it of $\mathrm{Mn}_{1.08} \mathrm{Bi}$ alloy are formed at $360^{\circ} \mathrm{C}(630 \mathrm{~K})$. If the $\mathrm{Mn}_{1.08} \mathrm{Bi}$ was formed, magnetic force was not lost due to ferromagnetism. Surface modification of diamond (110) with manganese at $450^{\circ} \mathrm{C}$ in vacuum then heating at $270^{\circ} \mathrm{C}$ for 5 minutes in vacuum with bismuth produces $\mathrm{MnBi}$ on surface of diamond surface. It gives $100 \mu \mathrm{T}$ magnetic flux density in partially could move diamond grains by Neodymium magnet.

Using Hall effect sensor and VSM, the spontaneous magnetization with a surface magnetic flux density of $232 \mu \mathrm{T}$ was measured. The diamond (110) sample of which weight was $0.030 \mathrm{~g}$, modified with about $0.010 \mathrm{~g}$ of $\mathrm{MnBi}$ was lifted between the two solenoid coil controlled using a Hall effect sensor.

\section{Acknowledgements}

This research was supported by the Center for Advanced Materials Analysis at Tokyo Institute of Technology for X-ray Diffraction and VSM measurements. The research was also supported by the Japan Society for Promotion of Science KAKENHI (23656452).

\section{Conflicts of Interest}

The authors declare no conflicts of interest regarding the publication of this paper. 


\section{References}

[1] Onogi, T, Koyama, K. and Watanabe, K. (2007) Magnetic Properties of MnBi in High Magnetic Fields and High Temperature. Journal of the Japan Institute of Metals, 71, 489-493. https://doi.org/10.2320/jinstmet.71.489

[2] Karen, P., Kjekshus, A., Anderson, A.F. and Fjellvag, H. (1991) On the Phase Relations Structural Relations and Structural and Magnetic Properties of the Stable Manganese Carbide, $\mathrm{Mn}_{23} \mathrm{C}_{6}, \mathrm{Mn}_{5} \mathrm{C}_{2}, \mathrm{Mn}_{7} \mathrm{C}_{3}$. Acta Chemica Scandinavia, 45, 540-557. https://doi.org/10.3891/acta.chem.scand.45-0549

[3] Yamazaki, T. and Ninomiya, R. (2017) Magnet Formation by the Surface Modification of Diamond with Manganese Detected by the Magnetic Flux Density on the Surface. Materials Sciences and Applications, 8, 642-648.

https://doi.org/10.4236/msa.2017.88045

[4] Pandey, B., Das, D. and Kar, A.K. (2015) Electrical and Magnetic Properties of Electrodeposited Nickel Incorporated Diamond-Like Carbon Thin Films. Applied Surface Science, 337, 195-207. https://doi.org/10.1016/j.apsusc.2015.02.092

[5] Yoshii, S. (1972) Magneto-Optical Effects of MnBi Thin Film. Oyobutsuri, 41, 11221128.

[6] Zhang, X.D., Mehrtash, M. and Khamesee, M.B. (2015) Dual-Axial Motion Control of a Magnetic Leviation System Using Hall-Effect Sensors. IEEE ASME Transactions on Mechatronics, 1-11. 